VERSITA

Journal of Applied Mathematics, Statistics and Informatics (JAMSI), 8 (2012), No. 2

\title{
FRACTIONAL INTEGRAL INEQUALITIES FOR DIFFERENTIABLE CONVEX MAPPINGS AND APPLICATIONS TO SPECIAL MEANS AND A MIDPOINT FORMULA
}

\author{
CHUN ZHU, MICHAL FEČKAN AND JINRONG WANG
}

\begin{abstract}
In this paper, Riemann-Liouville type fractional integral identity and inequality for differentiable convex mappings are studied. Some applications to special means of real numbers are given. Finally, error estimates for a midpoint formula are also obtained.
\end{abstract}

Mathematics Subject Classification 2000: 26A33, 26A51, 26D15

Additional Key Words and Phrases: Fractional integral inequalities, Differentiable convex mappings, Special means, Midpoint formula.

\section{INTRODUCTION}

For $f \in L[a, b]$, the Riemann-Liouville integrals $J_{a+}^{\alpha} f$ and $J_{b-}^{\alpha} f$ of order $\alpha>0$ with $a \geq 0$ are defined by

$$
J_{a+}^{\alpha} f(x)=\frac{1}{\Gamma(\alpha)} \int_{a}^{x}(x-t)^{\alpha-1} f(t) d t, x>a,
$$

and

$$
J_{b-}^{\alpha} f(x)=\frac{1}{\Gamma(\alpha)} \int_{x}^{b}(t-x)^{\alpha-1} f(t) d t, x<b,
$$

respectively, where $\Gamma(\cdot)$ is the Gamma function and $J_{a+}^{0} f(x)=J_{b-}^{0} f(x)=f(x)$.

Fractional calculus and its widely application have recently been paid more and more attentions. For more recent development on fractional calculus, one can see the monographs of Baleanu et al. [1], Diethelm [2], Kilbas et al. [3], Lakshmikantham et al. [4], Miller and Ross [5], Michalski [6], Podlubny [7] and Tarasov [8].

It is remarkable that Sarikaya et al. [9] initial give the following interesting integral inequalities of Hermite-Hadamard type involving Riemann-Liouville fractional integrals.

THEOREM 1.1. Let $f:[a, b] \rightarrow R$ be a positive function with $0 \leq a<b$ and

The first and third authors acknowledge the support by the National Natural Science Foundation of China (11201091) and Key Projects of Science and Technology Research in the Chinese Ministry of Education (211169). The second author acknowledges the support by VEGA-MS 1/0507/11 and APVV-0134-10.

DOI 10.2478/v10294-012-0011-5

CUniversity of SS. Cyril and Methodius in Trnava 


\section{CHUN ZHU, MICHAL FEČKAN AND JINRONG WANG}

$f \in L[a, b]$. If $f$ is a convex function on $[a, b]$, then the following inequality for fractional integrals hold

$$
f\left(\frac{a+b}{2}\right) \leq \frac{\Gamma(\alpha+1)}{2(b-a)^{\alpha}}\left[J_{a^{+}}^{\alpha} f(b)+J_{b^{-}}^{\alpha} f(a)\right] \leq \frac{f(a)+f(b)}{2} .
$$

Clearly, if we put $\alpha=1$ in Theorem 1.1, then inequality (1) becomes to the following known Hermite-Hadamard's inequality:

$$
f\left(\frac{a+b}{2}\right) \leq \frac{1}{b-a} \int_{a}^{b} f(x) d x \leq \frac{f(a)+f(b)}{2} .
$$

For more recent results which generalize, improve, and extend the inequalities presented above, one can see Abramovich et al. [10], Cal et al. [11], Avci et al. [12], Ödemir et al. [13; 14], Dragomir [15; 16], Sarikaya et al. [17], Xiao et al. [18], Xi et al. [19], Bessenyei [20], Tseng et al. [21], Niculescu [22] and references therein.

Unfortunately, Sarikaya et al. [9] only give some results connected with the right part of the equality (1). Some pioneer works connected with the left part of the equality (2) have been reported by Kirmaci and Ödemir [23; 24]. However, some related results connected with the left part of the equality (1) have not been studied extensively.

In the present paper, we will investigate some inequalities connected with the left part of the equality (1). In order to achieve our goals, we have to establish a important fractional integral identity (see Lemma 2.1) for differentiable convex mappings via Riemann-Liouville fractional integrals, which will be widely used to derive a inequality for connected with the left part of the equality (1) for differentiable convex mappings (see Theorem 2.3). We also give some applications to special means of real numbers and obtain error estimates for a midpoint formula.

\section{FRACTIONAL INTEGRAL IDENTITY AND INEQUALITY FOR DIFFERENTIABLE CONVEX MAPPINGS}

We establish a important fractional integral identity for differentiable convex mappings.

Lemma 2.1. Let $f:[a, b] \rightarrow \mathbb{R}$ be a differentiable mapping on $(a, b)$ with $a<b$. If $f^{\prime} \in L[a, b]$, then the following equality for fractional integrals holds:

$$
\begin{aligned}
& \frac{\Gamma(\alpha+1)}{2(b-a)^{\alpha}}\left[J_{a+}^{\alpha} f(b)+J_{b-}^{\alpha} f(a)\right]-f\left(\frac{a+b}{2}\right) \\
= & \frac{b-a}{2}\left[\int_{0}^{1} k f^{\prime}(t a+(1-t) b) d t-\int_{0}^{1}\left[(1-t)^{\alpha}-t^{\alpha}\right] f^{\prime}(t a+(1-t) b) d t\right]
\end{aligned}
$$

where

$$
k= \begin{cases}1, & 0 \leq t<\frac{1}{2} \\ -1, & \frac{1}{2} \leq t<1\end{cases}
$$


Proof. It suffices to note that

$$
\begin{aligned}
I= & \int_{0}^{\frac{1}{2}} f^{\prime}(t a+(1-t) b) d t-\int_{\frac{1}{2}}^{1} f^{\prime}(t a+(1-t) b) d t \\
& -\int_{0}^{1}\left[(1-t)^{\alpha}-t^{\alpha}\right] f^{\prime}(t a+(1-t) b) d t \\
= & {\left[\int_{0}^{\frac{1}{2}} f^{\prime}(t a+(1-t) b) d t\right]+\left[-\int_{\frac{1}{2}}^{1} f^{\prime}(t a+(1-t) b) d t\right] } \\
& +\left[-\int_{0}^{1}(1-t)^{\alpha} f^{\prime}(t a+(1-t) b) d t\right]+\left[\int_{0}^{1} t^{\alpha} f^{\prime}(t a+(1-t) b) d t\right] \\
:= & I_{1}+I_{2}+I_{3}+I_{4} .
\end{aligned}
$$

Integrating by parts, we have

$$
\begin{aligned}
I_{1} & =\int_{0}^{\frac{1}{2}} f^{\prime}(t a+(1-t) b) d t=\left.\frac{1}{a-b} f(t a+(1-t) b)\right|_{0} ^{\frac{1}{2}} \\
& =\frac{1}{b-a}\left[-f\left(\frac{a+b}{2}\right)+f(b)\right], \\
I_{2} & =-\int_{\frac{1}{2}}^{1} f^{\prime}(t a+(1-t) b) d t=\left.\frac{-1}{a-b} f(t a+(1-t) b)\right|_{\frac{1}{2}} ^{1} \\
& =\frac{1}{b-a}\left[f(a)-f\left(\frac{a+b}{2}\right)\right] .
\end{aligned}
$$

Put $x=t a+(1-t) b$, we have

$$
\begin{aligned}
I_{3} & =-\int_{0}^{1}(1-t)^{\alpha} f^{\prime}(t a+(1-t) b) d t \\
& =-\left.\frac{(1-t)^{\alpha}}{a-b} f(t a+(1-t) b)\right|_{0} ^{1}-\frac{\alpha}{a-b} \int_{0}^{1}(1-t)^{\alpha-1} f(t a+(1-t) b) d t \\
& =-\frac{f(b)}{b-a}+\frac{\alpha}{b-a} \int_{b}^{a}\left(\frac{x-a}{b-a}\right)^{\alpha-1} \frac{f(x)}{a-b} d x \\
& =-\frac{f(b)}{b-a}+\frac{\Gamma(\alpha+1)}{(b-a)^{\alpha+1}} J_{b-}^{\alpha} f(a),
\end{aligned}
$$

and

$$
\begin{aligned}
I_{4} & =\int_{0}^{1} t^{\alpha} f^{\prime}(t a+(1-t) b) d t \\
& =\left.\frac{t^{\alpha}}{a-b} f(t a+(1-t) b)\right|_{0} ^{1}-\frac{\alpha}{a-b} \int_{0}^{1} t^{\alpha-1} f(t a+(1-t) b) d t \\
& =-\frac{f(a)}{b-a}+\frac{\alpha}{b-a} \int_{b}^{a}\left(\frac{b-x}{b-a}\right)^{\alpha-1} \frac{f(x)}{a-b} d x \\
& =-\frac{f(a)}{b-a}+\frac{\Gamma(\alpha+1)}{(b-a)^{\alpha+1}} J_{a+}^{\alpha} f(b) .
\end{aligned}
$$




\section{CHUN ZHU, MICHAL FEČKAN AND JINRONG WANG}

Submitting (5), (6), (7), (8) to (4), it follows that

$$
I=-\frac{2}{b-a} f\left(\frac{a+b}{2}\right)+\frac{\Gamma(\alpha+1)}{(b-a)^{\alpha+1}}\left[J_{a+}^{\alpha} f(b)+J_{b-}^{\alpha} f(a)\right] .
$$

Thus, by multiplying both sides by $\frac{b-a}{2}$, we have conclusion (3).

Remark 2.2. In Lemma 2.1, if we put $\alpha=1$ then the equality (3) becomes

$$
\begin{aligned}
& \frac{1}{b-a} \int_{a}^{b} f(x) d x-f\left(\frac{a+b}{2}\right) \\
= & \frac{b-a}{2}\left[\int_{0}^{\frac{1}{2}} f^{\prime}(t a+(1-t) b) d t-\int_{\frac{1}{2}}^{1} f^{\prime}(t a+(1-t) b) d t\right. \\
& \left.-\int_{0}^{1}(1-2 t) f^{\prime}(t a+(1-t) b) d t\right] .
\end{aligned}
$$

Using the above fractional integral identity, we can obtain the following result.

THEOREM 2.3. Let $f:[a, b] \rightarrow \mathbb{R}$ be a differentiable mapping on $(a, b)$ with $a<b$. If $\left|f^{\prime}\right|$ is convex on $[a, b]$, then the following inequality for fractional integrals holds:

$$
\begin{aligned}
& \left|\frac{\Gamma(\alpha+1)}{2(b-a)^{\alpha}}\left[J_{a+}^{\alpha} f(b)+J_{b-}^{\alpha} f(a)\right]-f\left(\frac{a+b}{2}\right)\right| \\
\leq & \frac{b-a}{4(\alpha+1)}\left(\alpha+3-\frac{1}{2^{\alpha-1}}\right)\left[\left|f^{\prime}(a)\right|+\left|f^{\prime}(b)\right|\right] .
\end{aligned}
$$

Proof. Using Lemma 2.1 and the convexity of $\left|f^{\prime}\right|$, we have

$$
\begin{aligned}
& \left|\frac{\Gamma(\alpha+1)}{2(b-a)^{\alpha}}\left[J_{a+}^{\alpha} f(b)+J_{b-}^{\alpha} f(a)\right]-f\left(\frac{a+b}{2}\right)\right| \\
\leq & \frac{b-a}{2}\left[\int_{0}^{\frac{1}{2}}\left|f^{\prime}(t a+(1-t) b)\right| d t+\int_{\frac{1}{2}}^{1}\left|f^{\prime}(t a+(1-t) b)\right| d t\right. \\
& \left.+\int_{0}^{1}\left|(1-t)^{\alpha}-t^{\alpha}\right|\left|f^{\prime}(t a+(1-t) b)\right| d t\right] \\
\leq & \frac{b-a}{2}\left[\int_{0}^{\frac{1}{2}}\left[t\left|f^{\prime}(a)\right|+(1-t)\left|f^{\prime}(b)\right|\right] d t+\int_{\frac{1}{2}}^{1}\left[t\left|f^{\prime}(a)\right|+(1-t)\left|f^{\prime}(b)\right|\right] d t\right. \\
& \left.+\int_{0}^{1}\left|(1-t)^{\alpha}-t^{\alpha}\right|\left|f^{\prime}(t a+(1-t) b)\right| d t\right] \\
\leq & \frac{b-a}{2}\left[\int_{0}^{\frac{1}{2}}\left[t\left|f^{\prime}(a)\right|+(1-t)\left|f^{\prime}(b)\right|\right] d t+\int_{\frac{1}{2}}^{1}\left[t\left|f^{\prime}(a)\right|+(1-t)\left|f^{\prime}(b)\right|\right] d t\right. \\
& +\int_{0}^{\frac{1}{2}}\left[(1-t)^{\alpha}-t^{\alpha}\right]\left[t\left|f^{\prime}(a)\right|+(1-t)\left|f^{\prime}(b)\right|\right] d t \\
& \left.+\int_{\frac{1}{2}}^{1}\left[t^{\alpha}-(1-t)^{\alpha}\right]\left[t\left|f^{\prime}(a)\right|+(1-t)\left|f^{\prime}(b)\right|\right] d t\right] \\
:= & \frac{b-a}{2}\left(K_{1}+K_{2}+K_{3}+K_{4}\right) .
\end{aligned}
$$


After some calculation, we obtain

$$
\begin{aligned}
K_{1}= & \int_{0}^{\frac{1}{2}}\left[t\left|f^{\prime}(a)\right|+(1-t)\left|f^{\prime}(b)\right|\right] d t=\left.\frac{\left|f^{\prime}(a)\right|}{2} t^{2}\right|_{0} ^{\frac{1}{2}}+\left.\left|f^{\prime}(b)\right|\left(t-\frac{t^{2}}{2}\right)\right|_{0} ^{\frac{1}{2}} \\
= & \frac{1}{8}\left|f^{\prime}(a)\right|+\frac{3}{8}\left|f^{\prime}(b)\right|, \\
K_{2}= & \int_{\frac{1}{2}}^{1}\left[t\left|f^{\prime}(a)\right|+(1-t)\left|f^{\prime}(b)\right|\right] d t=\left.\frac{\left|f^{\prime}(a)\right|}{2} t^{2}\right|_{\frac{1}{2}} ^{1}+\left.\left|f^{\prime}(b)\right|\left(t-\frac{t^{2}}{2}\right)\right|_{\frac{1}{2}} ^{1} \\
= & \frac{3}{8}\left|f^{\prime}(a)\right|+\frac{1}{8}\left|f^{\prime}(b)\right|, \\
K_{3}= & \int_{0}^{\frac{1}{2}}\left[(1-t)^{\alpha}-t^{\alpha}\right]\left[t\left|f^{\prime}(a)\right|+(1-t)\left|f^{\prime}(b)\right|\right] d t \\
= & \left|f^{\prime}(a)\right|\left[\int_{0}^{\frac{1}{2}} t(1-t)^{\alpha} d t-\int_{0}^{\frac{1}{2}} t^{\alpha+1} d t\right] \\
& +\left|f^{\prime}(b)\right|\left[\int_{0}^{\frac{1}{2}}(1-t)^{\alpha+1} d t-\int_{0}^{\frac{1}{2}}(1-t) t^{\alpha} d t\right] \\
= & \left|f^{\prime}(a)\right|\left[\frac{1}{(\alpha+1)(\alpha+2)}-\frac{\left(\frac{1}{2}\right)^{\alpha+1}}{\alpha+1}\right]+\left|f^{\prime}(b)\right|\left[\frac{1}{(\alpha+2)}-\frac{\left(\frac{1}{2}\right)^{\alpha+1}}{\alpha+1}\right],
\end{aligned}
$$

and

$$
\begin{aligned}
K_{4}= & \int_{\frac{1}{2}}^{1}\left[t^{\alpha}-(1-t)^{\alpha}\right]\left[t\left|f^{\prime}(a)\right|+(1-t)\left|f^{\prime}(b)\right|\right] d t \\
= & \left|f^{\prime}(a)\right|\left[\int_{\frac{1}{2}}^{1} t^{\alpha+1} d t-\int_{\frac{1}{2}}^{1} t(1-t)^{\alpha} d t\right] \\
& +\left|f^{\prime}(b)\right|\left[\int_{\frac{1}{2}}^{1}(1-t) t^{\alpha} d t-\int_{\frac{1}{2}}^{1}(1-t)^{\alpha+1} d t\right] \\
= & \left|f^{\prime}(a)\right|\left[\frac{1}{(\alpha+2)}-\frac{\left(\frac{1}{2}\right)^{\alpha+1}}{\alpha+1}\right]+\left|f^{\prime}(b)\right|\left[\frac{1}{(\alpha+1)(\alpha+2)}-\frac{\left(\frac{1}{2}\right)^{\alpha+1}}{\alpha+1}\right] .
\end{aligned}
$$

Submitting (12), (13), (14), (15) to (11), we obtain the inequality (10). This completes the proof.

Remark 2.4. If we take $\alpha=1$ in Theorem 2.3, then the equality (10) becomes to the following inequality:

$$
\left|\frac{1}{b-a} \int_{a}^{b} f(x) d x-f\left(\frac{a+b}{2}\right)\right| \leq \frac{3(b-a)}{8}\left(\left|f^{\prime}(a)\right|+\left|f^{\prime}(b)\right|\right) .
$$

\section{APPLICATIONS TO SPECIAL MEANS AND A MIDPOINT FORMULA}

Consider the following special means (see Pearce and Pečarić [25]) for arbitrary real numbers $\alpha, \beta, \alpha \neq \beta$ as follows: 


\section{CHUN ZHU, MICHAL FEČKAN AND JINRONG WANG}

(i) $H(\alpha, \beta)=\frac{2}{\frac{1}{\alpha}+\frac{1}{\beta}}, \alpha, \beta \in \mathbb{R} \backslash\{0\}$,

(ii) $A(\alpha, \beta)=\frac{\alpha+\beta}{2}, \alpha, \beta \in \mathbb{R}$,

(iii) $L(\alpha, \beta)=\frac{\beta-\alpha}{\ln |\beta|-\ln |\alpha|},|\alpha| \neq|\beta|, \alpha \beta \neq 0$,

(iv) $L_{n}(\alpha, \beta)=\left[\frac{\beta^{n+1}-\alpha^{n+1}}{(n+1)(\beta-\alpha)}\right]^{\frac{1}{n}}, n \in \mathbb{Z} \backslash\{-1,0\}, \alpha, \beta \in \mathbb{R}, \alpha \neq \beta$.

Now, using the obtained results in Section 2, we give some applications to special means of real numbers.

Proposition 3.1. Let $a, b \in \mathbb{R}, a<b, 0$ does not belong to $[a, b]$ and $n \in \mathbb{Z}$, $|n| \geq 2$. Then

$$
\left|L_{n}^{n}(a, b)-A^{n}(a, b)\right| \leq \frac{3}{4}|n|(b-a) A\left(|a|^{n-1},|b|^{n-1}\right) .
$$

Proof. Applying Remark 2.4 for $f(x)=x^{n}$, one can obtain the result immediately.

Proposition 3.2. Let $a, b \in \mathbb{R}, a<b, 0$ does not belong to $[a, b]$. Then

$$
\left|L^{-1}(a, b)-A^{-1}(a, b)\right| \leq \frac{3}{4}(b-a) A\left(|a|^{-2},|b|^{-2}\right) .
$$

Proof. The assertion follows from Remark 2.4 applied for $f(x)=\frac{1}{x}$.

Proposition 3.3. Let $a, b \in \mathbb{R} \backslash\{0\}, a<b, a^{-1}>b^{-1}, 0$ does not belong to $[a, b]$ and $n \in \mathbb{Z},|n| \geq 2$. Then we have

$$
\left|L_{n}^{n}\left(b^{-1}, a^{-1}\right)-H^{-n}(b, a)\right| \leq \frac{3}{4}|n|\left(a^{-1}-b^{-1}\right) H^{-1}\left(|a|^{n-1},|b|^{n-1}\right),
$$

and

$$
\left|L^{-1}\left(b^{-1}, a^{-1}\right)-H(b, a)\right| \leq \frac{3}{4}\left(a^{-1}-b^{-1}\right) H^{-1}\left(|a|^{-2},|b|^{-2}\right) .
$$

Proof. Making the substitutions $a \rightarrow b^{-1}, b \rightarrow a^{-1}$ in the inequalities (17) and (18), one can obtain desired inequalities (19) and (20) respectively, where $A^{-1}\left(a^{-1}, b^{-1}\right)=H(a, b)=\frac{2}{\frac{1}{a}+\frac{1}{b}}, b^{-1}<a^{-1}$.

To end this paper, we give an application to a midpoint formula. As in Pearce and Pečarić [25], let $d$ be a division $a=x_{0}<x_{1}<\cdots<x_{n-1}<x_{n}=b$ of the interval $[a, b]$ and consider the quadrature formula

$$
\int_{a}^{b} f(x) d x=T(f, d)+E(f, d),
$$

where

$$
T(f, d)=\sum_{i=0}^{n-1} f\left(\frac{x_{i}+x_{i+1}}{2}\right)\left(x_{i+1}-x_{i}\right) .
$$

is the midpoint version and $E(f, d)$ denotes the approximation error. Here, we derive some error estimates for midpoint formula. 
THEOREM 3.4. Let $f:[a, b] \rightarrow \mathbb{R}$ be a differentiable mapping on $(a, b)$ with $a<b$. If $\left|f^{\prime}\right|$ is convex on $[a, b]$, then in (21), for every division d of $[a, b]$, we have

$$
|E(f, d)| \leq \frac{3}{8} \sum_{i=0}^{n-1}\left(x_{i+1}-x_{i}\right)^{2}\left(\left|f^{\prime}\left(x_{i}\right)\right|+\left|f^{\prime}\left(x_{i+1}\right)\right|\right) .
$$

Proof. Applying Remark 2.4 on the subinterval $\left[x_{i}, x_{i+1}\right](i=0,1, \cdots, n-1)$ of the division $d$, we get

$\left|\int_{x_{i}}^{x_{i+1}} f(x) d x-f\left(\frac{x_{i}+x_{i+1}}{2}\right)\left(x_{i+1}-x_{i}\right)\right| \leq \frac{3}{8}\left(x_{i+1}-x_{i}\right)^{2}\left(\left|f^{\prime}\left(x_{i}\right)\right|+\left|f^{\prime}\left(x_{i+1}\right)\right|\right)$.

Summing over $i$ from 0 to $n-1$ and taking into account that $\left|f^{\prime}\right|$ is convex, we obtain, by the triangle inequality, that

$$
\begin{aligned}
\left|\int_{a}^{b} f(x) d x-T(f, d)\right| & =\left|\sum_{i=0}^{n-1}\left[\int_{x_{i}}^{x_{i+1}} f(x) d x-f\left(\frac{x_{i}+x_{i+1}}{2}\right)\left(x_{i+1}-x_{i}\right)\right]\right| \\
& \leq \sum_{i=0}^{n-1}\left|\int_{x_{i}}^{x_{i+1}} f(x) d x-f\left(\frac{x_{i}+x_{i+1}}{2}\right)\left(x_{i+1}-x_{i}\right)\right| \\
& \leq \frac{3}{8} \sum_{i=0}^{n-1}\left(x_{i+1}-x_{i}\right)^{2}\left(\left|f^{\prime}\left(x_{i}\right)\right|+\left|f^{\prime}\left(x_{i+1}\right)\right|\right) .
\end{aligned}
$$

The proof is completed.

\section{REFERENCES}

[1] D. Baleanu, J. A. T. Machado, A.C.-J. Luo, Fractional dynamics and control, Springer, 2012.

[2] K. Diethelm, The analysis of fractional differential equations, Lecture Notes in Mathematics, 2010.

[3] A. A. Kilbas, H. M. Srivastava, J. J. Trujillo, Theory and applications of fractional differential equations, Elsevier Science B.V., 2006.

[4] V. Lakshmikantham, S. Leela, J. Vasundhara Devi, Theory of fractional dynamic systems, Cambridge Scientific Publishers, 2009.

[5] K. S. Miller, B. Ross, An introduction to the fractional calculus and differential equations, John Wiley, 1993.

[6] M. W. Michalski, Derivatives of noninteger order and their applications, Dissertationes Mathematicae, CCCXXVIII, Inst. Math., Polish Acad. Sci., 1993.

[7] I. Podlubny, Fractional differential equations, Academic Press, 1999.

[8] V. E. Tarasov, Fractional dynamics: Application of fractional calculus to dynamics of particles, fields and media, Springer, HEP, 2011.

[9] M. Z. Sarikaya, E. Set, H. Yaldiz, N. Başak, Hermite-Hadamard's inequalities for fractional integrals and related fractional inequalities, Math. Comput. Model., (2012), doi:10.1016/j.mcm.2011.12.048.

[10] S. Abramovich, J. Barić, Josip Pečarić, Fejer and Hermite-Hadamard type inequalities for superquadratic functions, J. Math. Anal. Appl., 344(2008), 1048-1056.

[11] J. Cal, J. Carcamob, L. Escauriaza, A general multidimensional Hermite-Hadamard type inequality, J. Math. Anal. Appl., 356(2009), 659-663.

[12] M. Avci, H. Kavurmaci, M. E. Ödemir, New inequalities of HermiteCHadamard type via s-convex functions in the second sense with applications, Appl. Math. Comput., 217(2011), 5171-5176. 


\section{CHUN ZHU, MICHAL FEČKAN AND JINRONG WANG}

[13] M. E. Ödemir, M. Avci, E. Set, On some inequalities of Hermite-Hadamard type via $m$ convexity, Appl. Math. Lett., 23(2010), 1065-1070.

[14] M. E. Ödemir, M. Avci, H. Kavurmaci, Hermite-Hadamard-type inequalities via $(\alpha, m)$ convexity, Comput. Math. Appl., 61(2011), 2614-2620.

[15] S. S. Dragomir, Hermite-Hadamard's type inequalities for operator convex functions, Appl. Math. Comput., 218(2011), 766-772.

[16] S. S. Dragomir, Hermite-Hadamard's type inequalities for convex functions of selfadjoint operators in Hilbert spaces, Linear Algebra Appl., 436(2012), 1503-1515.

[17] M. Z. Sarikaya, N. Aktan, On the generalization of some integral inequalities and their applications, Math. Comput. Model., 54(2011), 2175-2182

[18] Z. Xiao, Z. Zhang, Y. Wu, On weighted Hermite-Hadamard inequalities, Appl. Math. Comput., 218(2011), 1147-1152.

[19] B. Xi, R. Bai, F. Qi, Hermite-Hadamard type inequalities for the $m$-and $(\alpha, m)$ geometrically convex functions, Aequat. Math., (2012) doi:10.1007/s00010-011-0114-x.

[20] M. Bessenyei, The Hermite-Hadamard inequality in Beckenbach's setting, J. Math. Anal. Appl., 364(2010), 366-383.

[21] K. Tseng, S. Hwang, K. Hsu, Hadamard-type and Bullen-type inequalities for Lipschitzian functions and their applications, Comput. Math. Appl., (2012), doi:10.1016/j.camwa.2011.12.076.

[22] C. P. Niculescu, The Hermite-Hadamard inequality for log-convex functions, Nonlinear Anal.:TMA, 75(2012), 662-669.

[23] U. S. Kirmaci, Inequalities for differentiable mappings and applications to special means of real numbers and to midpoint formula, Appl. Math. Comput., 147(2004), 137-146.

[24] U. S. Kirmaci, M. E. Özdemir, On some inequalities for differentiable mappings and applications to special means of real numbers and to midpoint formula, Appl. Math. Comput., 153(2004), 361-368.

[25] C. E. M. Pearce, J. E. Pečarić, Inequalities for differentiable mappings with application to special means and quadrature formula, Appl. Math. Lett., 13(2000), 51-55.

Chun Zhu,

Department of Mathematics,

Guizhou University

Guiyang, Guizhou 550025, P.R. China

e-mail: czhumath@126.com

\section{Michal Fečkan}

Department of Mathematical Analysis and Numerical Mathematics

Faculty of Mathematics, Physics and Informatics

Comenius University, Mlynská dolina,

84248 Bratislava, Slovakia

e-mail: Michal.Feckan@fmph.uniba.sk

JinRong Wang

Department of Mathematics

Guizhou University

Guiyang, Guizhou 550025, P.R. China

e-mail: wjr9668@126.com; sci.jrwang@gzu.edu.cn 